\title{
CLASSIFICATION OF TENSOR PRODUCT IMMERSIONS WHICH ARE OF 1-TYPE
}

\author{
by BANG-YEN CHEN
}

(Received 9 November, 1992)

1. Introduction. Let $V$ and $W$ be two vector spaces over the field of real numbers R. Then we have the notion of the tensor product $V \otimes W$. If $V$ and $W$ are inner product spaces with their inner products given respectively by $\langle,\rangle_{V}$ and $\langle,\rangle_{w}$, then $V \otimes W$ is also an inner product space with inner product defined by

$$
\langle v \otimes w, x \otimes y\rangle=\langle v, x\rangle_{V}\langle w, y\rangle_{w} \quad \forall v \otimes w, x \otimes y \in V \otimes W .
$$

Let $E^{m}$ denote the $m$-dimensional Euclidean space with the canonical Euclidean inner product. Then, with respect to the inner product defined above, $E^{m} \otimes E^{\prime \prime \prime}$ is isometric to $E^{m m}$. By applying this algebraic notion, we have the notion of tensor product map $f \otimes h: M \rightarrow E^{\prime \prime} \otimes E^{m^{\prime}} \equiv E^{m m^{\prime}}$ associated with any two maps $f: M \rightarrow E^{\prime \prime \prime}$ and $h: M \rightarrow E^{\prime^{\prime}}$ of a given Riemannian manifold $(M, g)$ defined as follows:

$$
(f \otimes h)(p)=f(p) \otimes h(p) \in E^{m} \otimes E^{m^{\prime}} \quad \forall p \in M
$$

Denote by $\mathscr{R}(M)$ the set of all transversal immersions from an $n$-dimensional Riemannian manifold $(M, g)$ into Euclidean spaces; i.e., immersions $f: M \rightarrow E^{\prime \prime}$ with $f(p) \notin T_{*}\left(T_{p} M\right)$ for $p \in M$. Then $\otimes$ is a binary operation on $\mathscr{R}(M)$. Hence, if $f: M \rightarrow E^{\prime \prime}$ and $h: M \rightarrow E^{\prime \prime}$ are immersions belonging to $\mathscr{R}(M)$, then their tensor product map $f \otimes h: M \rightarrow E^{m} \otimes E^{\prime \prime \prime}$ is an immersion in $\mathscr{R}(M)$, called the tensor product immersion of $f$ and $h$.

A smooth map $x: M \rightarrow E^{\prime \prime}$ from a Riemannian manifold $M$ into a Euclidean $m$-space $E^{\prime \prime}$ is said to be of finite type ( $k$-type) if it can be decomposed into a sum of finitely many ( $k$, not counting a constant function) eigenfunctions of the Laplacian $\Delta$ of $M$ from different eigenspaces, i:e., we have

$$
x=x_{0}+x_{1}+\ldots+x_{k},
$$

where $x_{0}$ is a constant vector and $\Delta x_{t}=\lambda_{t} x_{t}, t=1,2, \ldots, k$, where $\lambda_{1}, \ldots, \lambda_{k}$ are $k$ different eigenvalues of the Laplacian of $M$ and $\Delta$ acts on a vector function componentwise. In that case we have $P(\Delta)\left(x-x_{0}\right)=0$, where $P(T)=\prod_{i=1}^{k}\left(T-\lambda_{i}\right)$. If $x$ cannot be represented as a finite sum (1.3), it is said to be of infinite type. The notions of finite type maps and finite type submanifolds were introduced about a decade ago (for instance [2], [7]). Since then many interesting results in this respect have been obtained by various authors. In particular, it has been proved that many important submanifolds are of finite type. Of course, the simplest finite type submanifolds are the 1-type submanifolds. A well-known result of $\mathrm{T}$. Takahashi [6] asserts that 1-type submanifolds are precisely those that are minimal in a hypersphere or minimal in the ambient Euclidean space.

Since the 1-type maps and 1-type submanifolds are the simplest maps and the simplest submanifolds in the finite type theory, it is fundamental to classify 1-type maps and 1-type submanifolds. For this reason, we shall investigate the tensor product immersion $f \otimes h$ associated with any two isometric immersions $f: M \rightarrow E^{\prime \prime}$ and $h: M \rightarrow E^{m^{\prime}}$ in $\mathscr{R}(M)$ in this article. As a result, we can completely determine all tensor product immersions $f \otimes h$ which are of 1-type. 
Because the tensor product immersion $f \otimes f$ associated with a single isometric immersion $f: M \rightarrow E^{m}$ is nothing but the quadric representation studied in [4] by I. Dimitric. Our result provides a vast generalization of the main result of [4].

2. Preliminaries. Let $f: M \rightarrow E^{m}$ be a map from an $n$-dimensional Riemannian manifold $(M, g)$ into the Euclidean $m$-space $E^{m}$. Denote by $\nabla$ and $\tilde{\nabla}$ the Levi-Civita connections of $(M, g)$ and of Euclidean spaces, respectively. The second fundamental form $\sigma_{f}$ and the energy density $e(f)$ of the map $f$ are then given respectively by

$$
\begin{gathered}
\sigma_{f}(X, Y)=\tilde{\nabla}_{X}\left(f_{*} Y\right)-f_{*}\left(\nabla_{X} Y\right), \\
e(f)=\frac{1}{2}\|d f\|^{2} .
\end{gathered}
$$

If $f: M \rightarrow E^{m}$ is an isometric immersion, the second fundamental form $\sigma_{f}$ is a normal-bundle-valued bilinear map on $T(M) \times T(M)$. The energy $E(f)$ of $f$ is defined by $E(f)=\int_{M} e(f) d V$. The Euler-Lagrange operator associated with $E$ shall be written as $\tau(f)=\operatorname{div}(d f)$ and is known as the tension field of $f$.

For a map $f: M \rightarrow E^{m}$, one has

$$
\Delta f=-\tau(f), \quad \tau(f)=\text { trace }_{g} \sigma_{f} .
$$

If $f: M \rightarrow E^{m}$ is an isometric immersion, the mean curvature vector field $H_{f}$ of $f$ is given by $H_{f}=\frac{1}{n}$ trace $\sigma_{f}=\frac{1}{n} \tau(f)$. Let $A^{f}$ and $D^{f}$ denote the Weingarten map and the normal connection of the immersion $f$, respectively. A normal vector field $\xi$ of $f$ is said to be a parallel normal section if $D^{f \xi}=0$, identically. A normal vector field $\xi$ is said to be a umbilical section if the Weingarten map $A_{\xi}^{f}$ in the direction of $\xi$ is proportional to the identity map. The immersion $f: M \rightarrow E^{m}$ is said to be totally umbilical if its second fundamental form $\sigma_{f}$ satisfies $\sigma_{f}(X, Y)=\mu g(X, Y) \tau(f)$, for some function $\mu$ on $M$. The immersion $f$ is called totally geodesic if its second fundamental form $\sigma_{f}$ vanishes identically.

An immersion $f: M \rightarrow E^{m}$ is said to be spherical if $f(M)$ is contained in a hypersphere of $E^{m}$ centered at the origin of $E^{m}$.

Given two isometric immersions $f: M \rightarrow E^{m}$ and $h: M \rightarrow E^{m^{\prime}}$, their tensor product immersion $f \otimes h: M \rightarrow E^{m} \otimes E^{m^{\prime}}$ is not isometric, in general. In fact we have the following two propositions.

PROPOSITION 1. Let $f \in \mathscr{R}(M)$ be an isometric immersion of an $n$-dimensional Riemannian manifold $M$ into $E^{\prime \prime}$ with $n \geq 2$ and take a natural number $k \geq 2$. Then $f^{k}=f \otimes \ldots \otimes f(k$ times $)$ is conformal if and only if $f$ is spherical.

Proof. For any tangent vector $X \in T_{p} M$ we have

$$
\left(f^{k}\right)_{*}(X)=f_{*}(X) \otimes f^{k-1}(p)+f(p) \otimes f_{*}(X) \otimes f^{k-2}(p)+\ldots+f^{k-1}(p) \otimes f_{*}(X),
$$

where $f_{*}$ denotes the differential of $f$. Thus, for any $X, Y \in T_{p} M$, we have

$$
\begin{aligned}
\left\langle\left(f^{k}\right)_{*}(X),\left(f^{k}\right)_{*}(Y)\right\rangle= & k u(p)^{k-1}\left\langle f_{*}(X), f_{*}(Y)\right\rangle+k(k-1) u(p)^{k-2} \\
& \times\left\langle f_{*}(X), f(p)\right\rangle\left\langle f_{*}(Y), f(p)\right\rangle,
\end{aligned}
$$

where $u(p)=\langle f(p), f(p)\rangle$. For any given $X \in T_{p} M$, choose $Y \in T_{p} M$ perpendicular to $X$. If $f^{k}$ is conformal, then (2.4) yields

$$
0=\left\langle\left(f^{k}\right)_{*}(X),\left(f^{k}\right)_{*}(Y)\right\rangle=k(k-1) u(p)^{k-2}\left\langle f_{*}(X), f(p)\right\rangle\left\langle f_{*}(Y), f(p)\right\rangle,
$$


from which we may obtain $\left\langle f_{*}(X), f(p)\right\rangle\left\langle f_{*}(Y), f(p)\right\rangle=0$ because $f \in \mathscr{R}(M)$. Since this is true for any orthogonal $X, Y \in T_{p} M, p \in M$, the linearity and the continuity of $f_{*}$ implies that $\left\langle f_{*}(X), f(p)\right\rangle=0$ for any $X \in T_{p}(M), p \in M$. From this we conclude that $f(M)$ is contained in a hypersphere of $E^{m}$ centered at the origin; i.e. $f$ is spherical.

Conversely, if $f$ is spherical, then (2.4) implies that

$$
\left\langle\left(f^{k}\right)_{*}(X),\left(f^{k}\right)_{*}(Y)\right\rangle=k u(p)^{k-1}\left\langle f_{*}(X), f_{*}(Y)\right\rangle
$$

for any $X, Y \in T_{p} M$. This shows that $f^{k}$ is conformal.

Remark. For $k=2$, Proposition 1 is due to Dimitric [4].

Proposition 2. Let $f, h \in \mathscr{R}(M)$ be two isometric immersions from an $n$-dimensional $(n \geq 2)$ Riemannian manifold $M$ into Euclidean spaces $E^{m}$ and $E^{m^{\prime}}$, respectively. Then the tensor product immersion $f \otimes h: M \rightarrow E^{m} \otimes E^{m}$ is conformal if and only if locally either $f$ or $h$ is spherical.

Proof. For each tangent vector $X \in T_{p} M$, we have

$$
(f \otimes h)_{*}(X)=f(p) \otimes h_{*}(X)+f_{*}(X) \otimes h(p) .
$$

Thus we find

$$
\left\langle(f \otimes h)_{*}(X),(f \otimes h)_{*}(Y)\right\rangle=
$$

$$
(\langle f(p), f(p)\rangle+\langle h(p), h(p)\rangle)\langle X, Y\rangle+2\left\langle f(p), f_{*}(X)\right\rangle\left\langle h(p), h_{*}(X)\right\rangle .
$$

If $f$ is spherical on an open subset $\mathscr{V}$ of $M$, then $\left\langle f(p), f_{*}(X)\right\rangle=0$ for any $X \in T_{p} M$, $p \in \mathscr{V}$. Thus, by (2.6), $f \otimes h$ is conformal on $\mathscr{V}$. Therefore, $f \otimes h$ is conformal if locally either $f$ or $h$ is spherical.

Conversely, if $f \otimes h$ is conformal, then (2.6) implies that

$$
\left\langle f(p), f_{*}(X)\right\rangle\left\langle h(p), h_{*}(X)\right\rangle=\mu(p)\langle X, X\rangle
$$

for some function $\mu$ on $M$. Since $\operatorname{dim} M=n \geq 2$, there exists a unit vector $X \in T_{p} M$ such that $\left\langle f(p), f_{*}(X)\right\rangle=0$. Thus, by (2.7), $\mu=0$ which implies that either $\left\langle f(p), f_{*}(X)\right\rangle=0$ or $\left\langle h(p), h_{*}(X)\right\rangle=0$ for $X \in T_{p} M$. Hence, either $X(\langle f, f\rangle)=0$ or $X(\langle h, h\rangle)=0$. Assume that there is a vector $X \in T_{p} M$ such that $X(\langle f, f\rangle) \neq 0$, then $X(\langle f, f\rangle) \neq 0$ for any $X$ in some dense open subset $U$ of $T_{p} M$. Thus, by continuity, we get $d(\langle h, h\rangle)=0$ at $p$. Let $W=\{q \in M: d(\langle h, h\rangle)(q) \neq 0\}$. Then $W$ is an open subset of $M$ and we have $d(\langle f, f\rangle)=0$ on $W$. This implies that $f$ is locally spherical on $W$. From these we may conclude that if $f \otimes h$ is conformal, then locally either $f$ or $h$ is spherical.

Lemma 3. Let $f: M \rightarrow E^{m}$ and $h: M \rightarrow E^{m}$ be a totally geodesic isometric immersion and a minimal isometric immersion from an $n$-dimensional $(n \geq 2)$ Riemannian manifold $M$ into Euclidean spaces $E^{m}$ and $E^{m^{\prime}}$, respectively. Then their tensor product immersion $f \otimes h: M \rightarrow E^{m} \otimes E^{m^{\prime}}$ is of infinite type.

Proof. If $f$ is a totally geodesic isometric immersion, then $M$ is isometric to an open portion of Euclidean $n$-space. Thus $h$ is an isometric minimal immersion from a flat Riemannian manifold into $E^{m^{\prime}}$ which implies that $h$ is also totally geodesic by the 
equation of Gauss. Therefore, by direct computation, we may find

$$
\Delta(f \otimes h)=2 \sum_{i} f_{*}\left(e_{i}\right) \otimes h_{*}\left(e_{i}\right),
$$

where $e_{1}, \ldots, e_{n}$ is a local orthonormal frame field of the tangent bundle $T(M)$ of $M$. On the other hand, we have

$$
\begin{aligned}
\tilde{\nabla}_{X}\left(\sum_{i} f_{*}\left(e_{i}\right) \otimes h_{*}\left(e_{i}\right)\right)=\sum_{i} \sigma_{f}\left(e_{i}, X\right) \otimes h_{*}\left(e_{i}\right)+\sum_{i} f_{*}\left(e_{i}\right) \otimes \sigma_{h}\left(e_{i}, X\right) \\
+\sum_{i, j=1}^{n} \omega_{i}^{j}(X) f_{*}\left(e_{j}\right) \otimes h_{*}\left(e_{i}\right)+\sum_{i, j=1}^{n} \omega_{i}^{j}(X) f_{*}\left(e_{i}\right) \otimes h_{*}\left(e_{j}\right),
\end{aligned}
$$

where $\nabla_{X} e_{j}=\sum_{k=1}^{n} \omega_{j}^{k}(X) e_{k}$ and $\bar{\nabla}$ is the Levi-Civita connection of $E^{m} \otimes E^{m^{\prime}}$. Since $\omega_{j}^{i}=-\omega_{i}^{j}$, this implies

$$
\tilde{\nabla}_{X}\left(\sum_{i} f_{*}\left(e_{i}\right) \otimes h_{*}\left(e_{i}\right)\right)=\sum_{i} \sigma_{f}\left(e_{i}, X\right) \otimes h_{*}\left(e_{i}\right)+\sum_{i} f_{*}\left(e_{i}\right) \otimes \sigma_{h}\left(e_{i}, X\right)
$$

Because both $f$ and $h$ are totally geodesic, (2.9) yields

$$
\bar{\nabla}_{X}\left(\sum_{i} f_{*}\left(e_{i}\right) \otimes h_{*}\left(e_{i}\right)\right)=0
$$

From (2.10) we get

$$
\Delta^{\prime}(f \otimes h)=0, \quad l \geq 2 .
$$

Suppose $f \otimes h$ is of $k$-type (with finite $k$ ). Then there exists a polynomial $P=P(t)$ of degree $k$ and a constant vector $a$ in $E^{m} \otimes E^{m^{\prime}}$ such that $P(\Delta)(f \otimes h-a)=0$. Thus, by (2.8) and (2.11), there exists a constant $\mu$ such that

$$
\sum_{i} f_{*}\left(e_{i}\right) \otimes h_{*}\left(e_{i}\right)=\mu(f \otimes h-a)
$$

Since the left-hand side of $(2.12)$ is never zero, $\mu \neq 0$. By taking the covariant derivative of (2.12) with respect to $X \in T_{p} M$ and using (2.10) we find

$$
f_{*}(X) \otimes h_{*}(X)+f(p) \otimes h_{*}(X)=0
$$

which is impossible because $\operatorname{dim} M=n>1$. Therefore, $f \otimes h$ is of infinite type.

We mention the following result (Theorem 25 of [3]) for later use.

LEMMA 4. Let $f: M \rightarrow E^{m}$ and $h: M \rightarrow E^{m^{\prime}}$ be two spherical isometric immersions of a Riemannian manifold $M$ into Euclidean spaces $E^{m}$ and $E^{m^{\prime}}$, respectively. Then their tensor product immersion $f \otimes h$ is of 1-type if and only if $M$ is isometric to an open portion of an ordinary $n$-sphere and $f$ and $h$ are totally umbilical isometric immersions.

3. 1-type tensor product immersions. The purpose of this section is to prove the following classification theorem. 
THEOREM 5. Let $f, h \in \mathscr{R}(M)$ be two isometric immersions from an $n$-dimensional $(n \geq 2)$ Riemannian manifold $M$ into Euclidean spaces $E^{\prime \prime}$ and $E^{m^{\prime}}$, respectively. Then their tensor product immersion $f \otimes h: M \rightarrow E^{m} \otimes E^{m^{\prime}}$ is of 1-type if and only if both $f$ and $h$ are spherical isometric immersions which map $M$ into open portions of hyperspheres with the same radius in some $(n+1)$-dimensional linear subspaces of $E^{m}$ and $E^{m^{\prime}}$, respectively.

Proof. If $f \otimes h$ is of 1-type, then there exist a real number $\lambda$ and a constant vector $c \in E^{m} \otimes E^{m^{\prime}}$ such that

$$
\Delta(f \otimes h)=n \lambda(f \otimes h)-c .
$$

On the other hand, we have (cf. Lemma 16 of [3])

$$
\Delta(f \otimes h)=-n H_{f} \otimes h-n f \otimes H_{h}-2 \sum_{i} f_{*}\left(e_{i}\right) \otimes h_{*}\left(e_{i}\right),
$$

where $e_{1}, \ldots, e_{n}$ is a local orthonormal frame field of the tangent bundle $T(M)$ of $M$ and $H_{f}$ and $H_{h}$ are the mean curvature vector fields of $f$ and $h$, respectively.

Let $X$ be a tangent vector of $M$. Then, by taking the covariant derivative of both sides of (3.1) with respect to $X$ and by applying (2.9) and (3.2), we may obtain

$-n A_{H_{f}}^{f} X \otimes h-n f \otimes A_{H_{h}}^{h} X+n D_{X}^{f} H_{f} \otimes h+n f \otimes D_{X}^{h} H_{h}$

$$
\begin{aligned}
+n H_{f} & \otimes h_{*}(X)+n f_{*}(X) \otimes H_{h}+2 \sum_{i} \sigma_{f}\left(e_{i}, X\right) \otimes h_{*}\left(e_{i}\right) \\
& +2 \sum_{i} f_{*}\left(e_{i}\right) \otimes \sigma_{h}\left(e_{i}, X\right)+n \lambda f_{*}(X) \otimes h+n \lambda f \otimes h_{*}(X)=0,
\end{aligned}
$$

where $A^{f}, D^{f}, \sigma_{f}$ (respectively, $A^{h}, D^{h}, \sigma_{h}$ ) are the Weingarten map, the normal connection and the second fundamental form of $f: M \rightarrow E^{m}$ (respectively, of $h: M \rightarrow E^{m^{\prime}}$ ), respectively.

For the immersions $f: M \rightarrow E^{m}$ and $h: M \rightarrow E^{m^{\prime}}$ we also denote by $f$ and $g$ respectively the position vector fields of $f: M \rightarrow E^{m}$ and $h: M \rightarrow E^{m^{\prime}}$. Denote by $f^{\perp}, f^{T}$ (respectively, $h^{\perp}, h^{\mathrm{T}}$ ) the normal and the tangential components of $f$ (respectively, of $h$ ).

Let $U=\left\{p \in M: f^{\perp}(p)=0\right\}$. Assume that the interior $U^{()}$of $U$ is non-empty. Then $f=f^{\mathrm{T}}$ on $U^{0}$. For any vector $X$ tangent to $U^{0}$, we have

$$
\bar{\nabla}_{X} f^{\mathrm{T}}=\tilde{\nabla}_{X} f=f_{*}(X) .
$$

Hence, we get $\sigma_{f}\left(X, f_{*}^{-1}\left(f^{\mathrm{T}}\right)\right)=0$. Because $f \in \mathscr{R}(M), f=f^{\mathrm{T}} \neq 0$. Let $e_{1}$ be the unit tangent vector of $M$ such that $f^{\mathrm{T}}=\left|f^{\mathrm{T}}\right| f_{*}\left(e_{1}\right)$. Then (3.4) yields

$$
\sigma_{f}\left(X, e_{1}\right)=0, \quad \forall X \in T U^{\circ}
$$

which implies that $e_{1}$ is an eigenvector of $A_{\xi}^{f}$ for any normal vector $\xi$ of $f$ at any point in $U^{0}$.

By considering the (normal $\otimes$ tangential)-component of (3.3), we find

$$
n D_{X}^{f} H_{f} \otimes h^{\mathrm{T}}+n H_{f} \otimes h_{*}(X)+2 \sum_{i} \sigma_{f}\left(e_{i}, X\right) \otimes h_{*}\left(e_{i}\right)=0
$$

Let $\bar{e}_{1}, \ldots, \bar{e}_{n}$ be an orthonormal local frame on $U^{0}$ such that $h_{*}\left(\bar{e}_{1}\right)$ is in the direction of $h^{\mathrm{T}}$. Then (3.6) yields

$$
\sigma_{f}\left(\bar{e}_{1}, \bar{e}_{i}\right)=\sigma_{f}\left(\bar{e}_{i}, \bar{e}_{j}\right)=0, \quad n H_{f}+2 \sigma_{f}\left(\bar{e}_{i}, \bar{e}_{i}\right)=0, \quad i, j=2, \ldots, n ; \quad i \neq j .
$$


From (3.7) we may obtain

$$
\sigma_{f}\left(\bar{e}_{1}, \bar{e}_{1}\right)=\frac{n(n+1)}{2} H_{f}
$$

Because $\bar{e}_{1}, \ldots, \bar{e}_{n}$ are eigenvectors of $A_{\xi}^{f}$ for any normal vector $\xi,(3.5),(3.7)$ and (3.8) imply that $H_{f}=0$ on $U^{0}$. Thus

$$
\sigma_{f}=0
$$

on $U^{0}$, by (3.7) and (3.8). Therefore (3.3) yields

$-n f \otimes A_{H_{h}}^{h} X+n f \otimes D_{X}^{h} H_{h}+n f_{*}(X) \otimes H_{h}$

$$
+2 \sum_{i} f_{*}\left(e_{i}\right) \otimes \sigma_{h}\left(e_{i}, X\right)+n \lambda f_{*}(X) \otimes h+n \lambda f \otimes h_{*}(X)=0
$$

on $U^{0}$.

By considering the (tangential $\otimes$ tangential)-component of (3.10) we find

$$
-f \otimes A_{H_{h}}^{h} e_{i}+\lambda f_{*}\left(e_{i}\right) \otimes h^{\mathrm{T}}+\lambda f \otimes h_{*}\left(e_{i}\right)=0, \quad i=1, \ldots, n .
$$

Since $f_{*}\left(e_{1}\right)$ is in the direction of $f$, by taking the $f_{*}\left(e_{i}\right) \otimes h^{\mathrm{T}}$ component of (3.11) with $i \neq 1$, we find either $\lambda=0$ or $h^{\mathrm{T}}=0$ on $U^{0}$. If $\lambda=0$, then (3.11) yields $A_{H_{h}}^{h}=0$. Thus, $H_{h}=0$. This implies that the isometric immersion $h$ is minimal on $U^{0}$. Therefore, by applying Lemma 3 , we conclude that the tensor product immersion $f \otimes h$ is of infinite type on $U^{0}$ and so $f \otimes h$ is of infinite type which is a contradiction.

If $\lambda \neq 0$, then $h=h^{\perp}$ on $U^{\prime \prime}$. This implies that $h$ is spherical on each connected component of $U^{0}$. Thus, (3.11) yields

$$
-f \otimes A_{H_{h}}^{h} e_{i}+\lambda f \otimes h_{*}\left(e_{i}\right)=0
$$

which implies

$$
A_{H_{h}}^{h} X=\lambda h_{*}(X), \quad X \in T U^{\prime \prime} .
$$

In particular, this shows that $H_{h} \neq 0$. Consequently, (3.10) becomes

$$
n f \otimes D_{X}^{h} H_{h}+n f_{*}(X) \otimes H_{h}+2 \sum_{i=1}^{n} f_{*}\left(e_{i}\right) \otimes \sigma_{h}\left(e_{i}, X\right)+n \lambda f_{*}(X) \otimes h=0
$$

on $U^{0}$. Because $f_{*}\left(e_{1}\right)$ is in the direction of $f,(3.13)$ gives

$$
\begin{gathered}
\sigma_{h}\left(e_{j}, e_{j}\right)=-\frac{n}{2}\left(\lambda h+H_{h}\right), \quad j=2, \ldots, n, \\
\sigma_{h}\left(e_{i}, e_{j}\right)=0, \quad i \neq j, \quad i=1, \ldots, n, \quad j=2, \ldots, n .
\end{gathered}
$$

From (3.14) we find

$$
\sigma_{h}\left(e_{1}, e_{1}\right)=\frac{n}{2}\left\{(n-1) \lambda h+(n+1) H_{h}\right\}
$$

(3.14), (3.15) and (3.16) imply

$$
\operatorname{Im} \sigma_{h} \subset \operatorname{Span}\left\{h, H_{h}\right\}
$$


on $U^{0}$. Since $h=h^{\perp}$ on $U^{0}, \tilde{\nabla}_{X} h=h_{*}(X)$. Thus, the immersion $h: M \rightarrow E^{m^{\prime}}$ is also umbilical with respect to the normal direction $h^{\perp}=h$ on $U^{0}$. Combining this with (3.12) and (3.17) we conclude that $h: M \rightarrow E^{m^{\prime}}$ is totally umbilical on $U^{0}$. Because $H_{h} \neq 0$ on $U^{0}$, each connected component of $U^{0}$ is of positive constant sectional curvature. On the other hand, (3.9) implies that $U^{0}$ is flat which is a contradiction. Therefore, we conclude that $U$ is of measure zero. Thus, $f^{\perp} \neq 0$ almost everywhere on $M$. Similarly, $h^{\perp} \neq 0$ almost everywhere. Consequently, if $f \otimes h$ is of 1-type, then $f^{\perp} \neq 0$ and $h^{\perp} \neq 0$ on an open dense subset $V$ of $M$. For simplicity, we will work only on this open dense subset $V$ through the remaining part of the proof unless mentioned otherwise.

Now, we make the following claim

Claim 1. If $f^{\mathrm{T}}=0$ on an open subset $W$ of $V$, then $h^{\mathrm{T}}=0$ on $W$.

Assume Claim 1 is false. Then $h^{\mathrm{T}} \neq 0$ on $W$. Since $f^{\mathrm{T}}=0, f=f^{\perp}$. So, by considering the (tangential $\otimes$ tangential)-component of (3.3), we get

$$
A_{H_{f}}^{f} X \otimes h^{\mathrm{T}}=\lambda f_{*}(X) \otimes h^{\mathrm{T}}
$$

Because $h^{\mathrm{T}} \neq 0$ by assumption,

$$
A_{H_{f}}^{f} X=\lambda f_{*}(X) \text {. }
$$

By (3.18), we find $\left\langle H_{f}, H_{f}\right\rangle=\lambda$ which is a constant. Thus for any tangent vector $X$ we have $\left\langle D_{X}^{f} H_{f}, H_{f}\right\rangle=0$.

On the other hand, by considering the (tangential $\otimes$ normal)-component of (3.3) and using (3.18), we obtain

$$
n f_{*}(X) \otimes H_{h}+2 \sum_{i} f_{*}\left(e_{i}\right) \otimes \sigma_{h}\left(e_{i}, X\right)=0 .
$$

Thus

$$
n \delta_{i j} H_{h}+2 \sigma_{h}\left(e_{i}, e_{j}\right)=0, \quad i, j=1, \ldots, n
$$

from which we obtain $\sigma_{h}=0$, i.e. $h: M \rightarrow E^{m^{\prime}}$ is totally geodesic. Since $h$ is isometric, this implies $W$ is flat. Furthermore, we can assume that $H_{f} \neq 0$, since otherwise $f \otimes h$ is of infinite type by Lemma 3. From $\sigma_{h}=0,(3.3)$ and (3.18) we find

$$
n D_{X}^{f} H_{f} \otimes h+n H_{f} \otimes h_{*}(X)+2 \sum_{i} \sigma_{f}\left(e_{i}, X\right) \otimes h_{*}\left(e_{i}\right)+n \lambda f \otimes h_{*}(X)=0 .
$$

Now, let $\bar{e}_{1}, \ldots, \bar{e}_{n}$ be an orthonormal local frame field such that $h_{*}\left(\bar{e}_{1}\right)$ is in the direction of $h^{\mathrm{T}}$. Then, from (3.19), we may obtain

$$
\begin{gathered}
\sigma_{f}\left(\bar{e}_{i}, \bar{e}_{j}\right)=0, \quad i \neq j, \quad i, j=1, \ldots, n, \\
\sigma_{f}\left(\bar{e}_{j}, \bar{e}_{j}\right)=-\frac{n}{2}\left\{H_{f}+\lambda f\right\}, \quad j=2, \ldots, n .
\end{gathered}
$$

By (3.21) we find

$$
\sigma_{f}\left(\bar{e}_{1}, \bar{e}_{1}\right)=\frac{n(n+1)}{2} H_{f}+\frac{n(n-1)}{2} \lambda f
$$


From (3.20), (3.21) and (3.22) we know that the first normal space of $f: M \rightarrow E^{m}$ at each point $p \in W$ is spanned by $H_{f}(p)$ and $f(p)$. Since $f^{\mathrm{T}}=0, M$ is immersed in a hypersphere of $E^{m}$ centered at the origin. Thus the Weingarten map of the immersion $f$ at the normal direction $f^{\perp}=f$ is proportional to the identity map. Combining this with (3.18) we conclude that $f: M \rightarrow E^{m}$ is totally umbilical on $W$. Since $\sigma_{h}=0$ and both $f: M \rightarrow E^{m}$ and $h: M \rightarrow E^{m^{\prime}}$ are isometric, $f: M \rightarrow E^{m}$. is also totally geodesic on $W$. Hence, according to Lemma $3, f \otimes h$ is of infinite type which is a contradiction. Consequently, Claim 1 is proved.

Now we give the following.

Claim 2. There exist no non-empty open subset $V$ of $M$ such that $f^{\mathrm{T}} \neq 0$ on $V$.

In view of Claim 1, we may assume that $h^{\mathrm{T}} \neq 0$ on $V$, too. Furthermore, since $f^{\perp}$ and $h^{\perp}$ are nonzero almost everywhere on $M$, we may choose $V$ such that $f^{\perp}$ and $h^{\perp}$ are nonzero on $V$.

We choose orthonormal local frame fields $e_{n+1}, \ldots, e_{m}$ of the normal bundle $T_{f}^{\perp}(M)$ of $f: M \rightarrow E^{m}$ such that $e_{n+1}$ is in the direction of $f^{\perp}$ and choose orthonormal local frame fields $\bar{e}_{n+1}, \ldots, \bar{e}_{n}$ of $T \frac{1}{h}(M)$ such that $\bar{e}_{n+1}$ is in the direction of $h^{\perp}$.

Consider the (normal $\otimes$ normal)-component of (3.3); we find

$$
D_{X}^{f} H_{f} \otimes h^{\perp}+f^{\perp} \otimes D_{X}^{h} H_{h}=0
$$

from which we have

$$
D_{X}^{f} H_{f}=\alpha(X) f^{\perp}, \quad D_{X}^{h} H_{h}=\beta(X) h^{\perp},
$$

for some 1-forms $\alpha$ and $\beta$. Moreover, by considering the (tangential $\otimes$ tangential)component of (3.3) we may obtain

$$
\left(A_{H_{f}}^{f} X-\lambda f_{*}(X)\right) \otimes h^{\mathrm{T}}=f^{\mathrm{T}} \otimes\left(A_{H_{h}}^{h} X-\lambda h_{*}(X)\right)
$$

which implies

$$
A_{H_{f}}^{f} X=\lambda f_{*}(X)+\gamma(X) f^{\mathrm{T}}, \quad A_{H_{h}}^{h} X=\lambda h_{*}(X)+\delta(X) h^{\mathrm{T}}
$$

for some 1-forms $\gamma$ and $\delta$. From (3.24) we find

$$
\begin{gathered}
\gamma(X)\left\langle f, f_{*}(Y)\right\rangle=\gamma(Y)\left\langle f, f_{*}(X)\right\rangle, \\
\delta(X)\left\langle h, h_{*}(Y)\right\rangle=\delta(Y)\left\langle h, h_{*}(X)\right\rangle
\end{gathered}
$$

for tangent vectors $X, Y$. Therefore

$$
\gamma(X)=\delta(X)=0, \quad \text { if }\left\langle f_{*}(X), f^{\mathrm{T}}\right\rangle=0 .
$$

By considering the (tangent $\otimes$ normal)-component of (3.3) and using (3.23) and (3.24), we get

$$
n(\beta(X)-\gamma(X)) f^{\mathrm{T}} \otimes h^{\perp}+n f_{*}(X) \otimes H_{h}+2 \sum_{i} f_{*}\left(e_{i}\right) \otimes \sigma_{h}\left(e_{i}, X\right)=0 .
$$


Let $e_{1}, \ldots, e_{n}$ be an orthonomal local frame field of $M$ such that $f_{*}\left(e_{1}\right)$ is parallel to $f^{\mathrm{T}}$. From (3.26) we may obtain

$$
\sigma_{h}\left(e_{i}, e_{j}\right)=-\frac{n}{2} \delta_{i j} H_{h}, \quad i=1, \ldots, n, \quad j=2, \ldots, n .
$$

From (3.24), (3.25) and (3.27) we get

$$
\left\langle H_{h}, H_{h}\right\rangle=-\frac{2}{n} \lambda
$$

From (3.27) we have

$$
\sigma_{h}\left(e_{1}, e_{1}\right)=\frac{n(n+1)}{2} H_{h}
$$

(3.26) and (3.28) imply that the dimension of the first normal space is at most 1 and moreover $H_{h}=0$ if and only if $\sigma_{h}=0$. By using (3.25), (3.26), (3.27) and (3.29) we find

$$
\begin{gathered}
\beta(X)=0 \quad \text { if }\left\langle f_{*}(X), f^{\mathrm{T}}\right\rangle=0, \\
\left(\gamma\left(e_{1}\right)-\beta\left(e_{1}\right)\right) f^{\mathrm{T}} \otimes h^{\perp}=(n+2) f_{*}\left(e_{1}\right) \otimes H_{h} .
\end{gathered}
$$

(3.31) implies that $h^{\perp}$ is parallel to $H_{h}$.

Assume $H_{h} \neq 0$. Then $H_{h}=\alpha_{h} \bar{e}_{n+1}$ and $h^{\perp}=\mu \bar{e}_{n+1}$ for some functions $\alpha_{h}$ and $\mu$ on $M$. Let $Y$ be a tangent vector of $V$ such that $\left\langle f_{*}(Y), f^{\mathrm{T}}\right\rangle=0$. Then (3.25), (3.26), (3.27) and (3.29) yield

$$
n f_{*}(Y) \otimes H_{h}+2 \sum_{i} f_{*}\left(e_{i}\right) \otimes \sigma_{h}\left(Y, e_{i}\right)=0 .
$$

From (3.28), we find that $D_{e_{1}}^{h} H_{h}=\alpha_{h} D_{e_{1}}^{h} \bar{e}_{n+1}$ is perpendicular to the $h^{\perp}$. On the other hand, (3.23) shows that $D_{e_{1}}^{h} H_{h}$ is parallel to $h^{\perp}$. Therefore, $\beta\left(e_{1}\right)=0$. Combining this with (3.23) we find $D^{h} H_{h}=0$. Consequently, $V$ is locally immersed into an $(n+1)$-dimensional linear subspace $E^{n+1}$ of $E^{m^{\prime}}$ by $h$. Furthermore, from (3.27), (3.28) and (3.29), we know that $h(M)$ is a hypersurface of $E^{n+1}$ with two nonzero constant principal curvatures. But this is impossible by [5]. Therefore, $H_{h}=0$ on $V$. Hence, by (3.27) and (3.29), we obtain $\sigma_{h}=0$. Similarly, we have $\sigma_{f}=0$. But this is impossible according to Lemma 3 . Hence, $f^{\mathrm{T}}=0$ which contradicts the assumption. Consequently, Claim 2 is proved.

- From Claim 2 we know that if $f \otimes h$ is of 1-type, then $f^{\mathrm{T}}=h^{\mathrm{T}}=0$. This implies that both $f$ and $h$ are spherical isometric immersions. Hence, by applying Lemma 5, $M$ is isometric to an open portion of an ordinary $n$-sphere and both $f$ and $h$ are totally umbilical isometric immersions. Consequently, $M$ was immersed into open portions of hyperspheres with the same radii in some $(n+1)$-dimensional linear subspaces of $E^{m}$ and $E^{m^{\prime}}$, by $f$ and $h$, respectively.

The converse is already given by Lemma 4 .

\section{REFERENCES}

1. B. Y. Chen, Geometry of submanifolds (M. Dekker, 1973).

2. B. Y. Chen, Total mean curvature and submanifolds of finite type (World Scientific, 1984). 
3. B. Y. Chen, Differential geometry of semirings of immersions, I: General theory, Bull. Inst. Math. Acad. Sinica 21 (1993), 1-34.

4. I. Dimitric, Quadric representation of a submanifold, Proc. Amer. Math. Soc. 114 (1992), 201-210.

5. P. J. Ryan, Homogeneity and some curvature conditions for hypersurfaces, Tôhoku Math. J. (2) 21 (1969), 363-388.

6. T. Takahashi, Minimal immersions of Riemannian manifolds, J. Math. Soc. Japan 18 (1966), 380-385.

7. L. Verstraelen, On submanifolds of finite Chen type and of restricted type, Results Math. 20 (1991), 744-755.

Department of Mathematics

Michigan State University

EAST LANSING

Michigan 48824-1027, U.S.A. 